\title{
Fair success rates
}

\author{
For authors who have published in this journal, success rates of getting manuscripts peer-reviewed and \\ published do not correlate with submission history or academic seniority.
}

In informal conversations among academics, it is often said that more experienced researchers have it easier than their younger colleagues when it comes to getting published, in particular in highimpact journals. Certainly, seasoned researchers are likely to be more skilful at communicating their research effectively, and their previous achievements may be more widely known. Yet, when assessing manuscripts for publication, are editors and reviewers significantly biased by author names? We can't find any indication of favouritism in the data that we have gathered for 250 authors who have published in Nature Materials.

According to the data, for researchers who have published five or more research papers in this journal, the average author had a $50 \%$ success rate of having their submitted manuscripts sent out to external review, and a $27 \%$ success rate of getting their submissions published (deviations from the average can be significant, however; Fig. 1a). These success rates contrast with the roughly $15 \%$ peer-review rate and $5 \%$ accept rate typical of this journal (Nature Mater. 11, 743-744; 2012). The differences probably arise from the fact that authors who have published multiple papers in the journal are likely to have a better sense of the significance and quality of the papers that the journal publishes, and are also able to submit work that matches such expectations. Yet if the differences were to become significantly greater as a result of editors and reviewers favouring the most successful authors, this would be apparent when looking at individual success rates according to academic seniority and publication history in the journal.

We have not found evidence of any such bias. Success rates do not correlate with academic seniority or number of manuscripts submitted to the journal (Fig. 1a). For example, for the $50 \%$ of authors in the dataset who obtained their $\mathrm{PhD}$ degree in 1996 or earlier, the average success rate of getting manuscripts peerreviewed is $54 \%$; for the remainder $50 \%$ who obtained their degree after 1996, the corresponding rate is 58\%; and for the $20 \%$ of authors with $\mathrm{PhD}$ degrees from 2005 onwards, the average rate is $57 \%$. Differences
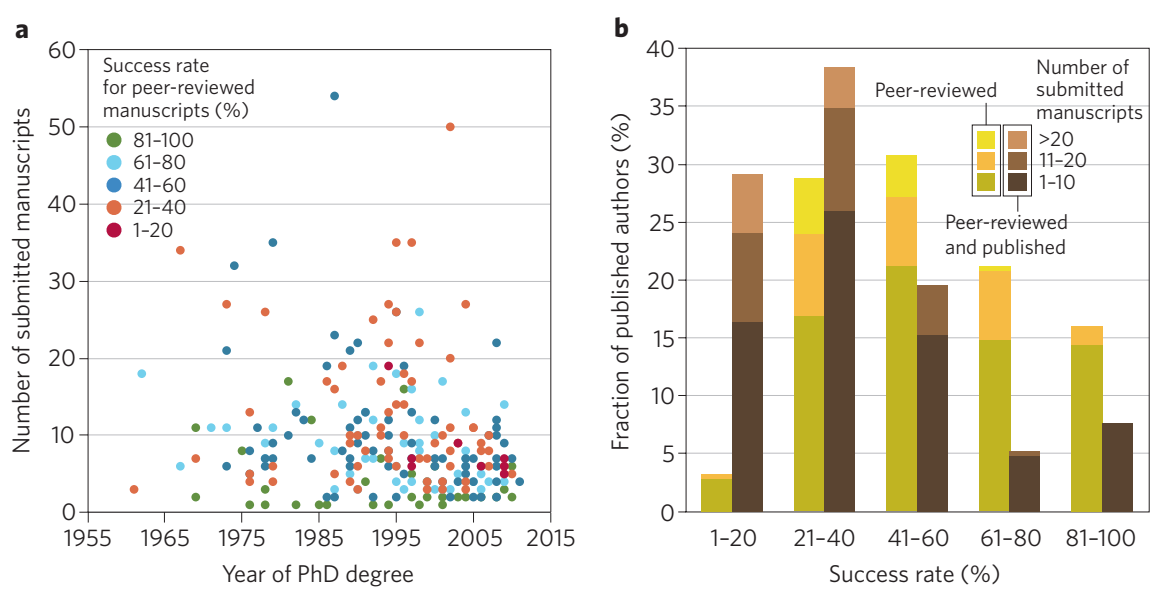

Figure 1 | Success rates of authors who have published in Nature Materials. a,b, Distribution of authors according to success rate of getting manuscripts peer-reviewed $(\mathbf{a}, \mathbf{b})$ and peer-reviewed and published (b); to number of submitted manuscripts (a,b); and to academic seniority (with year of PhD degree as a proxy; a). Data correspond to 250 randomly selected authors who are principal investigators and have published at least one paper in Nature Materials since 2013. For each author, number of submitted manuscripts and success rates include all the author's research manuscripts published or rejected (with or without peer review) by the journal since its launch in September 2002. In a, when more than one dot falls in the same position, the colour of the dot shown corresponds to that of the lowest success rate.

between these means are not statistically significant (the standard deviations are, respectively, 22\%, 26\% and 25\%).

Similarly, for the approximately $41 \%, 21 \%$ and $9 \%$ of authors who have published in the journal and submitted, respectively, 5-10, 11-20 and more than 20 manuscripts, the average success rates of getting manuscripts peer-reviewed are, respectively, 50\%, 51\% and $41 \%$ (with standard deviations of $20 \%, 19 \%$ and $12 \%$ ). Only differences with the latter group are significant (at a 95\% confidence level). The most likely explanation for this is that the group with the highest number of submitted manuscripts (lightest-yellow bar segments in Fig. 1b) includes a higher fraction of authors who are less self-selective about the manuscripts they submit to this journal. It is important to note that, because of simple arithmetics, authors who have submitted fewer than five papers have comparatively higher success rates - for example, $6 \%$ of 250 authors have submitted only one manuscript and thus have a success rate of $100 \%$ (the cluster of green dots at the bottom of Fig. 1a).
For the success rates of getting papers published, the differences between the groups are also not significant (except, again, for comparisons with the group of authors who have submitted more than 20 manuscripts). Expectedly, the numbers are lower $(39 \pm 26 \%$ and $33 \pm 22 \%$, respectively, for the pre-1996 and post-1996 groups, and $29 \pm 15 \%, 28 \pm 15 \%$ and $19 \pm 9 \%$ for the 5-10, 11-20 and $>20$ groups). In addition, the authorship pool is naturally skewed towards younger authors, who have submitted fewer manuscripts (41\% of authors obtained their $\mathrm{PhD}$ degree after 1996 and have sent 10 or fewer manuscripts to the journal; Fig. 1a).

Overall, about $31 \%$ of the authors who have published in Nature Materials have had a fair success rate, $40-60 \%$, of getting manuscripts past the editor (Fig. 1b). Yet the fraction of authors that got $40-60 \%$ of their submitted manuscripts published decreases to $19 \%$. Are these numbers fair? Perhaps the only thing we are sure of is that they are a consequence of the bias of editors and reviewers towards solid, highly significant work. 